\title{
Development of a Novel and Customizable Two-Solution Mixing Type Spray Nozzle for One-Step Preparation of Nanoparticle-Containing Microparticles
}

\author{
Tetsuya Ozeki, ${ }^{*}, a$ Yusuke Akiyama, ${ }^{b}$ Norimitsu Takahashi, ${ }^{b}$ Tatsuaki Tagami, ${ }^{a}$ Toshiyuki Tanaka, \\ Masashi Fujii, ${ }^{c}$ and Hiroaki Okada ${ }^{b}$ \\ ${ }^{a}$ Drug Delivery and Nano Pharmaceutics, Graduate School of Pharmaceutical Sciences, Nagoya City University; \\ 3-1 Tanabe-dori, Mizuho-ku, Nagoya, Aichi 467-8603, Japan: ${ }^{b}$ Department of Pharmaceutics and Drug Delivery, \\ School of Pharmacy, Tokyo University of Pharmacy and Life Science; 1432-1 Horinouchi, Hachioji, Tokyo 192-0392, \\ Japan: and ${ }^{c}$ Ohkawara Kakohki Co., Ltd.; 3847 Ikonobe-cho, Tsuzuki-ku, Yokohama, Kanagawa 224-0053, Japan. \\ Received March 26, 2012; accepted August 17, 2012
}

Production of drug nanoparticles is an effective strategy to enhance solubility and oral absorption of water-insoluble drugs. The handling of drug nanoparticles has been an important issue in drug formulation because nanoparticles easily aggregate each other and redispersion of these particles is very difficult. In the present study, we developed a unique two-solution mixing type spray nozzle that can prepare drug nanoparticles in microparticles in one step without any common solvent and surfactant, and then, the prepared formulation were evaluated. Ethylcellulose (EC) and mannitol (MAN) were used as a model polymer of water-insoluble compound and a water-soluble carrier, respectively. We characterized the EC/MAN microparticles produced by the novel spray nozzle when customizing the nozzle parts to mix EC and MAN solution. Relatively smaller EC nanoparticles $(<110 \mathrm{~nm})$ in MAN microparticles (approximately $3 \mu \mathrm{m})$ were obtained by changing the customizable parts in the nozzle. In addition, the core of EC nanoparticles $(<50 \mathrm{~nm})$ was also observed by atomic force microscopy. We also found that the mixing time in the nozzle parts affected the size and the standard deviation of EC nanoparticles. These results suggest that the size of EC nanoparticles in MAN microparticles is controllable by using this unique nozzle. After all, we could prepare MAN microparticles containing EC nanoparticles in one step by using the novel nozzle. The drug/MAN microparticles formulation produced by the nozzle may be useful for the handling of drug nanoparticles.

Key words two-solution mixing type nozzle; spray drier; composite particle; anti-solvent effect; one-step preparation

Improvement of water-insoluble drugs has been an important issue as increasing the promising drug candidates. The use of nano-sized drugs is an effective strategy to enhance drug solubility. ${ }^{1-5)}$ Several methods have been extensively employed to prepare smaller particles from large drug particles, such as the use of ball and jet mills, crystallization by evaporating the drug in an organic solvent, and preparation of emulsions and liposomes. Although these methods have advantages and disadvantages, the handling of nano-sized drug powders has been an important factor. Nanoparticles can easily aggregate with each other because of their great surface energy; hence, redispersion of these aggregates is very difficult and the effective properties derived from nanoparticles are lost. Therefore, technologies to preserve nanoparticles have been pursued.

In a previous study, we developed a four-fluid nozzle spray dryer to produce microparticles containing nano-sized drug particles. ${ }^{6-11)}$ This nozzle has two liquid and two gas passages that allow two compounds (e.g., a water-insoluble drug in organic solvent and a water-soluble carrier in aqueous solution) to be dissolved in separate solvents, thereby overcoming the problems of finding and using a common solvent. Although the detailed mechanism of the production of drug nanoparticles/water-soluble carrier microparticles by using the four-fluid nozzle is still unclear, we proposed a tentative mechanism. After the drug-organic solvent solution and the aqueous solution in a chamber with high-speed compressed air collide at the end of the nozzle, a powerful shockwave is generated

The authors declare no conflict of interest. at that spot, and accordingly, the solutions are atomized into droplets. These droplets are mixed and the drug begins to crystallize and form its core through the "anti-solvent effect," which means the effect of nano-sized drug crystallization by decreasing the solubility after the drug solvent is mixed with other drug-insoluble solvent. ${ }^{12-14)}$ We have previously utilized this nozzle to prepare drug/microparticle formulations for inhalation $^{6-8)}$ as a dry powder and to prepare two-drug composite microparticles ${ }^{9,11)}$ and microspheres containing polymeric nanoparticles. ${ }^{10)}$

The four-fluid spray nozzle was useful to produce the aforementioned drug nanoparticle-containing microparticle formulation. However, elaborate optimization was sometimes required to control and adjust the particle size of the formulation. Determining the optimal spray drying condition was a lengthy process as the drug-organic solution and aqueous solutions was were mixed outside the nozzle and the mixing time was expected to be short. In addition, it was still unclear whether an anti-solvent effect could be efficiently and homogenously induced by using the four-fluid nozzle.

In this study, we developed a two-solution mixing type nozzle for the simple production of microparticles containing drug nanoparticles (Fig. 1). This nozzle had a mixing component to mix the drug-organic solvent and aqueous solution in its nozzle. It was expected that water-insoluble drugs could begin to form a nano-sized drug-core in this component through the anti-solvent effect and that drug nanoparticles in microparticles could be effectively produced. In this study, we investigated whether this two-solution mixing type nozzle could 
produce smaller drug nanoparticles in microparticles by using ethylcellulose (EC) and mannitol (MAN) as a water-insoluble polymer and water-soluble carriers, respectively. Furthermore, the mixing component may be essential to control the size of drug particles. This component of the nozzle has four parts (injection tubes, mixing joint, mixing tube, and orifice), and several of them are customizable. The effect of customizing the mixing component in the nozzle was also investigated.

\section{MATERIALS AND METHODS}

Materials EC (EC-7, N-7 grade; molecular weight= 58000) was provided by Shin-Etsu Chemical Co., Ltd. (Tokyo, Japan). MAN was purchased from Wako Pure Chemical Industries, Ltd. (Osaka, Japan). All reagents were of analytical grade.

Preparation of EC/MAN Microparticles by Spray Dryer Equipped with a Spray Nozzle

Spray Dryer Spray dried particles were prepared using a spray dryer, Twin Jetter NL-5, (Ohkawara Kakohki Co., Ltd., Kanagawa, Japan). An overview of this dryer is presented in Supplementary Fig. 1 and on our homepage (Ohkawara Kakohki Co., Ltd. Site. http://www.oc-sd.co.jp/english/index. html). The spray dryer contains nine components (sample solution, compressed air, air insert, heater, spray nozzle, drying, cyclone, filter section, and drain parts).

Two-Solution Mixing Type Spray Nozzle (Type I Nozzle) An overview of the Type 1 nozzle (Ohkawara Kakohki Co., Ltd.) which was a prototype of the Type II nozzle is illustrated in Supplementary Fig. 2. The drug-organic solvent solution (EC solution) and the aqueous solution (MAN solution) pass through different liquid passages of the nozzle. In addition, this nozzle contains a mixing component to mix the EC and MAN solutions, after which EC-core particles begin to form in the solution through the anti-solvent effect in this component. The mixing solution is transported to the end of the nozzle and propelled at a high speed by atomizing air (mixing section). The sprayed mist collided at a spot (collision section) after which the EC/MAN mist containing micron-sized EC/MAN particles was generated. The collision energy was expected to be utilized to drive microparticle formation. The generated EC/MAN mist was then dried using heated air, and the dried EC/MAN microparticles were collected as a sample.

Spray-Drying Conditions of the EC/MAN Microparticles with the Type I Nozzle To produce EC/MAN microspheres, EC and MAN were first dissolved in separate solvents. EC was dissolved in $2 / 1$ acetone/methanol $(\mathrm{v} / \mathrm{v})$ at a final concentration of $0.278 \% \mathrm{w} / \mathrm{v}$, and MAN $(2.78 \% \mathrm{w} / \mathrm{v})$ was dissolved in water. Although the mixing ratio of $\mathrm{EC} /$ MAN was typically $1 / 10(\mathrm{w} / \mathrm{w})$, other ratios of EC/MAN $(1 / 5.7,1 / 7.7,1 / 22.8$, and $1 / 60.5, \mathrm{w} / \mathrm{w})$ were created by changing the flow rates of the EC and MAN solutions. Spray-drying of

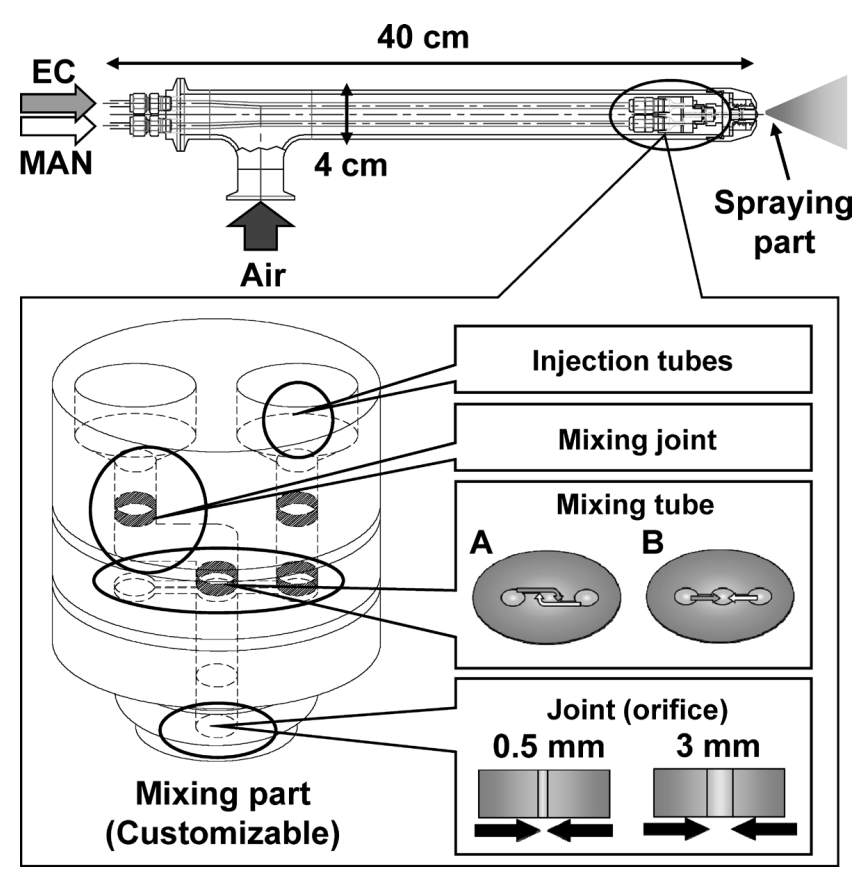

Fig. 1. Schematic Illustration of a Two-Solution Mixing Type II Spray Nozzle for One-Step Preparation of Drug-Containing Microspheres

EC/MAN microparticles was performed under the following conditions: inlet temperature, $60-80^{\circ} \mathrm{C}$; outlet temperature, $32-41^{\circ} \mathrm{C}$; and spray air pressure, $0.1 \mathrm{MPa}$.

Two-Solution Mixing Type II Spray Nozzle (Type II Nozzle) An overview of the Type II nozzle (Ohkawara Kakohki Co., Ltd.) is illustrated in Fig. 1. Unlike the Type I nozzle, the mixing component of this nozzle can be customized to mix the EC and MAN solutions. The EC and MAN solutions pass through different liquid passages of the nozzle. This nozzle contains a mixing component to mix the two solutions after which EC-core particles begin to form in the solution through the anti-solvent effect in this part of the nozzle as observed for the Type I nozzle. The mixing component of the Type II nozzle consists of four parts (injection tubes, mixing joint, mixing tube, and orifice), and accordingly, as shown in Table 1, combinations of the mixing components were compared in the present study after which the EC/MAN microparticles generated after spray-drying through the Type II nozzle were characterized. After the solution passed through the mixing component, the solution was sprayed as described in 'Two-Solution Mixing Type Spray Nozzle (Type I Nozzle)' for the Type 1 nozzle.

The total mixing time until the mixture was sprayed decreased from 0.25 to $0.02 \mathrm{~s}$ by adding air into the injection tube simultaneously with the EC solution. The total mixing time increased from 0.25 to $3 \mathrm{~s}$ by shifting the mixing parts to

Table 1. The Combination of Mixing Parts in Type II Spray Nozzle

\begin{tabular}{|c|c|c|c|c|c|c|c|c|}
\hline & Lot. 1 & Lot. 2 & Lot. 3 & Lot. 4 & Lot. 5 & Lot. 6 & Lot. 7 & Lot. 8 \\
\hline Mixing joint & \multicolumn{4}{|c|}{ Non-equipped } & \multicolumn{4}{|c|}{ Equipped } \\
\hline Mixing tube & \multicolumn{2}{|c|}{ A } & \multicolumn{2}{|c|}{ B } & \multicolumn{2}{|c|}{ A } & \multicolumn{2}{|c|}{$\mathrm{B}$} \\
\hline Joint (orifice) & $\phi 0.5$ & $\phi 3.0$ & $\phi 0.5$ & $\phi 3.0$ & $\phi 0.5$ & $\phi 3.0$ & $\phi 0.5$ & $\phi 3.0$ \\
\hline
\end{tabular}



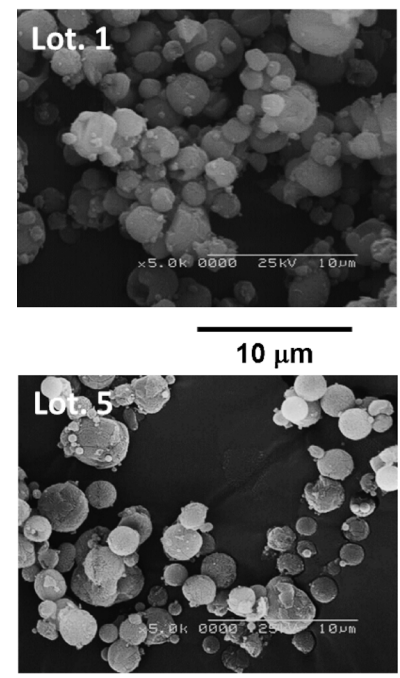

$10 \mu \mathrm{m}$

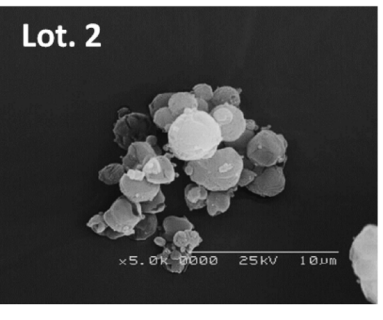

$10 \mu \mathrm{m}$

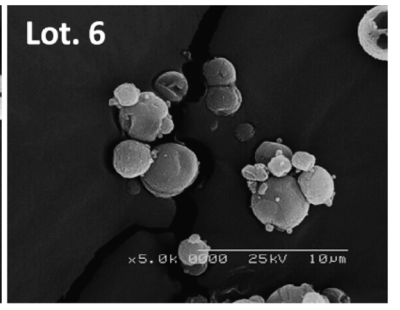

$10 \mu \mathrm{m}$

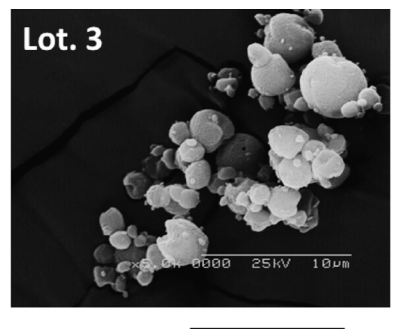

$10 \mu \mathrm{m}$

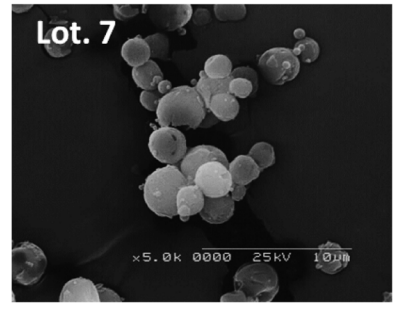

$10 \mu \mathrm{m}$

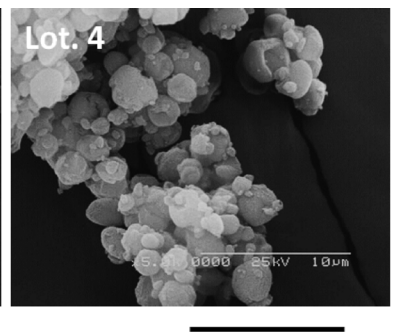

$10 \mu \mathrm{m}$

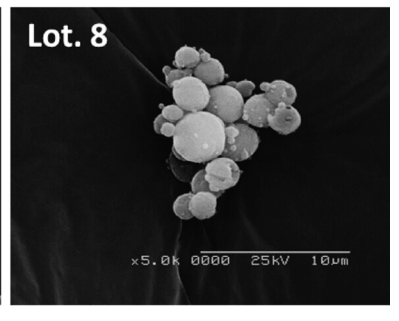

$10 \mu \mathrm{m}$

Fig. 2. SEM Photographs of EC/MAN Microparticles Using the Type II Nozzle with Various Combinations of Parts

the upper site ( $25 \mathrm{~cm}$; left side in Fig. 1).

Spray-Drying Conditions of EC/MAN Microparticles with the Type II Nozzle To produce EC/MAN microspheres, EC and MAN were first dissolved as described in 'Spray-Drying Conditions of the EC/MAN Microparticles with the Type I Nozzle' (the final ratio of EC/MAN was 1/10 $(w / w))$. Spray-drying of EC/MAN and EC/MAN microspheres was performed under the following conditions: inlet temperature, $115^{\circ} \mathrm{C}$; outlet temperature, $38-48^{\circ} \mathrm{C}$; and spray air pressure, $0.1 \mathrm{MPa}$.

Scanning Electron Microscopy (SEM) Images The particle structures of EC/MAN microparticles and EC nanoparticles were observed with an S-2250N scanning electron microscope (Hitachi, Tokyo, Japan) as previously described. $\left.{ }^{6}\right)$ In brief, EC/MAN particles were coated with 25 -nm-thick gold by using a quick carbon coater (SC-701; Sanyu Electronics, Tokyo, Japan), and these particles were then observed by SEM.

EC nanoparticles (dispersion) were obtained by dissolving the EC/MAN microparticles in ultrapure water. After filtration through a $0.1-\mu \mathrm{m}$ membrane filter, EC dispersion was spontaneously dried on the quick carbon coater, and the samples were coated with 25 -nm-thick gold. These particles were then observed by SEM.

Measurement of Mean Particle Diameter Measurements of EC/MAN microparticles and EC nanoparticles were performed as described previously. ${ }^{6}$ In brief, the mean particle diameters (horizontal Feret's diameter) of EC/MAN microparticles were determined. The mean particle diameter was defined as the median diameter of the cumulative curve of the number-basis particle size distribution.

The mean particle diameters of the EC dispersion was measured using a model DLS-7000 super dynamic light scattering spectrophotometer (Otsuka Electronics, Osaka, Japan) with excitation using an $\mathrm{Ar}$ laser at $5 \AA$ and $90^{\circ}$.

Atomic Force Microscopy (AFM) Images The size and surface morphology of EC nanoparticles prepared in 'Measurement of Mean Particle Diameter' were analyzed by AFM. The EC dispersion was filtered through a $0.05-\mu \mathrm{m}$ membrane filter. The sample solution was spontaneously dried and then observed by AFM (Nanoscope IV-D3100, Digital Instruments, Veeco, CA, U.S.A.) in tapping mode.

\section{RESULTS AND DISCUSSION}

Preparation of EC/MAN Microspheres Using a Type I Nozzle Spray Dryer We developed two-solution mixing type nozzles to produce water-insoluble drug-containing microparticles. The type I nozzle which served as a prototype for the Type II nozzle is shown in Supplementary Fig. 2. In the present study, EC, a hydrophobic polymer, was used and we attempted to produce EC/MAN microparticles. As shown in Supplementary Fig. 3, EC/MAN microparticles of various sizes were produced and SEM revealed that these microparticles were nearly spherical. The size of these microparticles slightly increased with an increase in the amount of MAN. The mean diameters of EC/MAN microparticles were 1.77 $(\mathrm{EC} / \mathrm{MAN}=1 / 5.7, \mathrm{w} / \mathrm{w}), 1.80(\mathrm{EC} / \mathrm{MAN}=1 / 7.7, \mathrm{w} / \mathrm{w}), 2.65(\mathrm{EC} /$ $\mathrm{MAN}=1 / 22.8, \mathrm{w} / \mathrm{w})$, and $3.48 \mu \mathrm{m}(\mathrm{EC} / \mathrm{MAN}=1 / 60.5, \mathrm{w} / \mathrm{w})$. The morphology of EC particles in MAN microparticles was evaluated by SEM after dissolving the particles in water. Nano-sized EC particles (approximately $200 \mathrm{~nm}$, spherical) were observed (data not shown). These results suggest that this two-solution type spray dryer could be feasible for the production of EC/MAN microparticles.

Preparation of EC/MAN Microspheres Using a Two-Solution Mixing Type Nozzle (Type II) Spray Dryer In the next phase of the study, we investigated a new spray nozzle (Type II) with customizable mixing components that can control the amount of EC and MAN solutions added to the mixture. The mixing component of the Type II nozzle contains four parts (injection tubes, mixing joint, mixing tube, and orifice) (Fig. 1), and several of them are customizable. In this study two types of mixing joint (bent or linear one), mixing tube (swirling or linear one) and orifice (short or long) were designed and then compared. We assumed that the slight difference of designed parts may affect the flowing rate, the surface area of solution and the pattern of collided solution, 

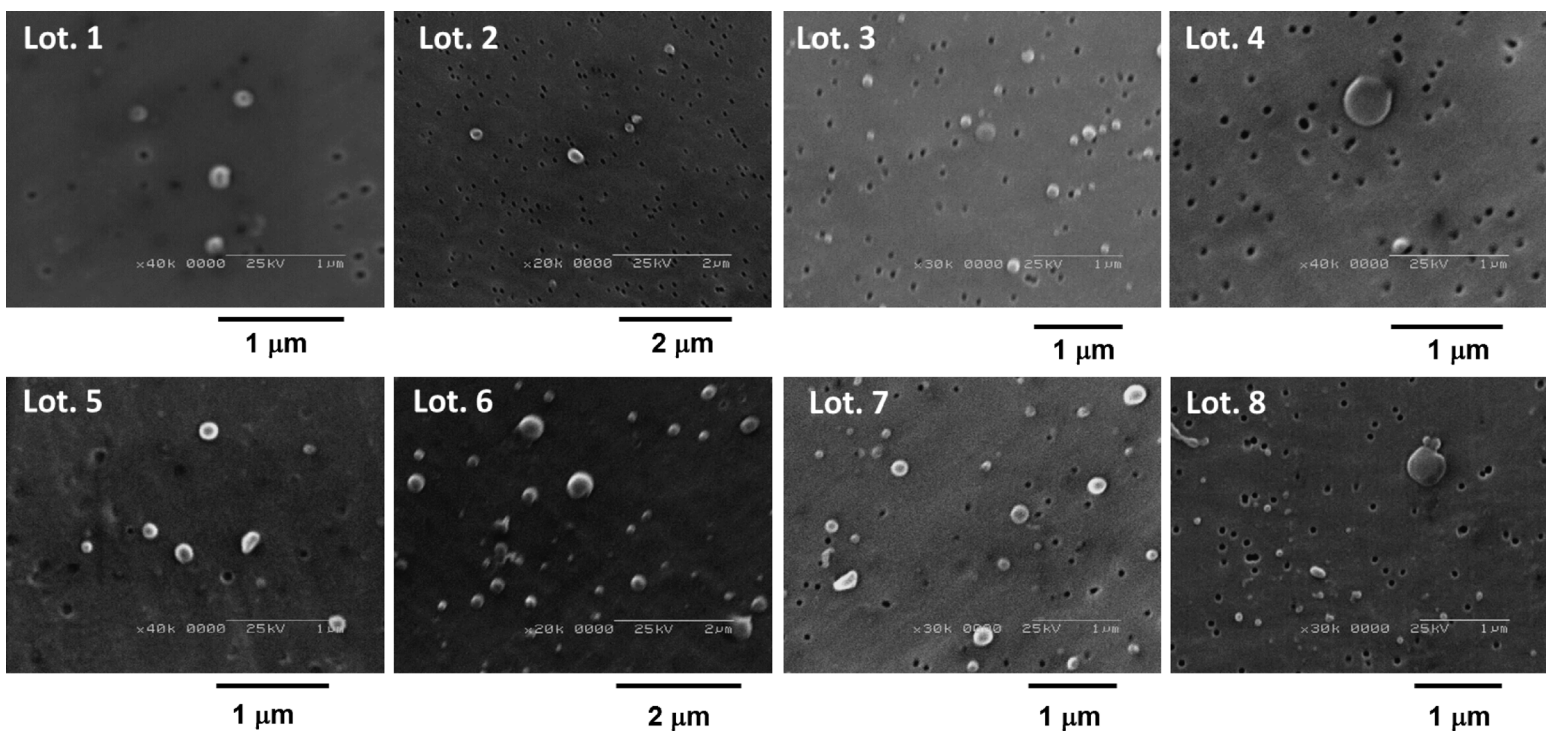

Fig. 3. SEM Photographs of EC Nanoparticles Using Various Combinations of Parts

EC nanoparticle suspensions were prepared as described in Materials and Methods.

resulting in the difference of particle size. In this study, optimization was performed by changing three parts (mixing joint, mixing tube, and orifice). The parts are illustrated in Fig. 1, and the combinations of parts are shown in Table 1. All combinations from Lot 1 to Lot 8 produced nearly spherical MAN microparticles (Fig. 2). The mean diameters of the microparticles produced using Lots $1-8$ were $2.25,2.77,3.14$, $3.05,3.24,3.37,3.39$, and $3.08 \mu \mathrm{m}$, respectively.

The EC particles in MAN microparticles were observed by SEM after dissolving the EC/MAN microparticles in water (Fig. 3). Nanoparticles were observed in all lots. The sizes of EC nanoparticles were confirmed using the dynamic light scattering method (Fig. 4). The mean diameter of the nanoparticles was approximately $100-200 \mathrm{~nm}$, although the mean diameters for Lots 5,6 , and 8 were relatively smaller $(<110 \mathrm{~nm})$. Thus, these results suggest that appropriate control of the flow route of EC and MAN solutions is critical to generating homogenous and smaller EC nanoparticles.

In our previous study using a four-fluid spray nozzle, it was difficult to obtain nanoparticles with such a small diameter $(<110 \mathrm{~nm})$. EC nanoparticles in MAN microparticles and poly(lactic-co-glycolic acid) (PLGA) nanoparticles in MAN exhibited diameters of 630 and $480 \mathrm{~nm}$ (submicron size, $<1 \mu \mathrm{m}$ ), respectively, ${ }^{10)}$ and rifampicin nanoparticles in MAN microparticles also exhibited diameters of approximately $200 \mathrm{~nm} .{ }^{6}$ By contrast, we succeeded in the preparation of relatively smaller EC nanoparticles $(<110 \mathrm{~nm})$ in MAN microparticles by using a two-solution mixing type nozzle (Type II) because of the difference in the structure of the nozzle. An organic drug solution and an aqueous solution were mixed inside the two-solution mixing type nozzle, whereas they were mixed outside in the four-solution nozzle. Therefore, the mixing conditions were remarkably different between these two nozzles. In addition, it was still unclear whether an anti-solvent effect could be efficiently and homogenously induced by using the four-fluid spray nozzle. Although it is difficult to compare the particles in the same spray-drying condition by using a Type II nozzle, the production of smaller

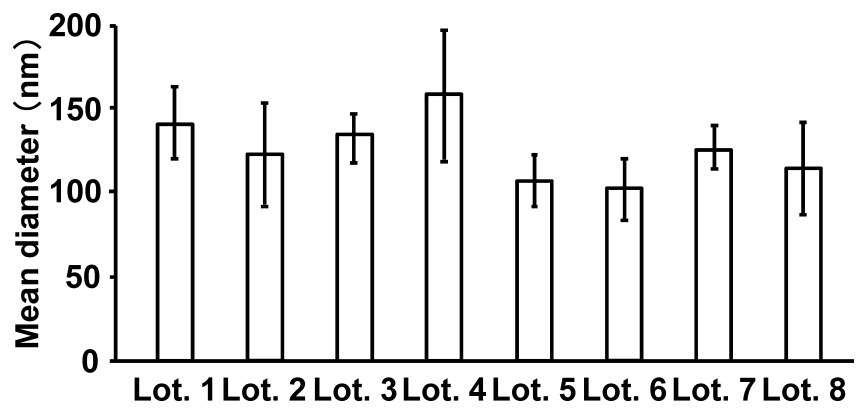

Fig. 4. Mean Diameters of EC Nanoparticles Measured by the Dynamic Light Scattering Method

EC nanoparticle suspensions were prepared as described in Materials and Methods.

nanoparticle-containing microparticles $(<110 \mathrm{~nm})$ is a novel point in this series of study. The smaller drug particles have advantages to enhance the dissolution rate and oral absorption. Our achievement would be utilized for the future study by using drug/MAN microparticles.

Next, the solution containing smaller EC nanoparticles was carefully observed by AFM. EC nanoparticles with diameters $<50 \mathrm{~nm}$ were observed after further filtration through a 0.05 $\mu \mathrm{m}$ filter membrane. As shown in Fig. 5, various sizes of EC nanoparticles were observed. These nanoparticles suggested that a nano-sized core began to form in the EC solution by means of the anti-solvent effect. These small EC-cores affect the small diameters.

Effect of the Mixing Time of Solutions in the Type II Nozzle on the Size of EC Nanoparticles We further customized the spray nozzle to alter the mixing time. To decrease the mixing time, the EC solution and air were co-injected into the injection tube. By contrast, to lengthen the mixing time, the mixing component was moved to the upper site (left side of the nozzle in Fig. 1) of the spray nozzle. The size of EC nanoparticles in MAN microparticles using different mixing times are shown in Fig. 6. When the mixing time was decreased $(0.02 \mathrm{~s})$, the mean diameter of EC nanoparticles was 
(A) Three dimension

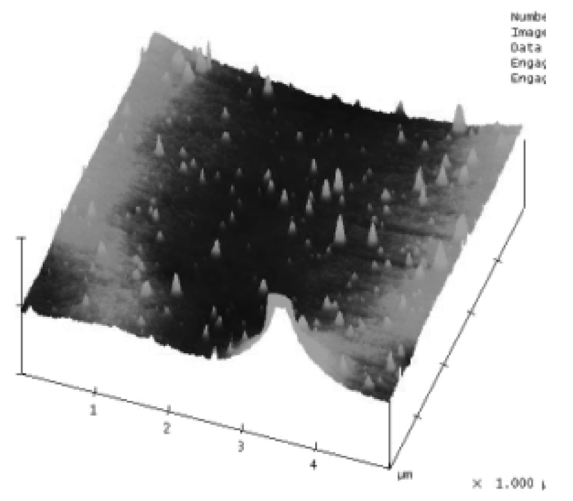

\section{(B) Two dimension}

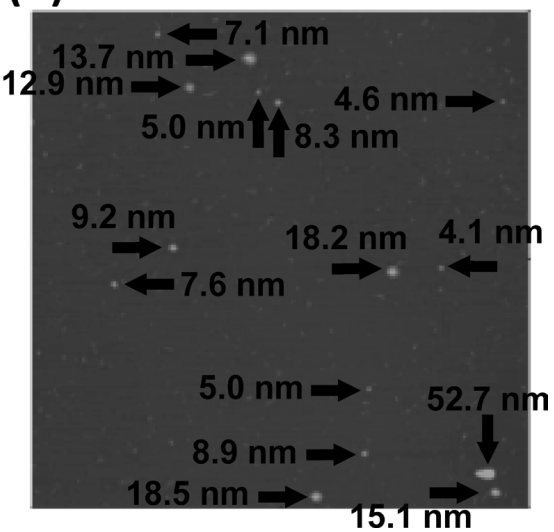

Fig. 5. AFM Images of EC Nanoparticles Using Various Combinations of Parts

EC nanoparticle suspensions were prepared as described in Materials and Methods.

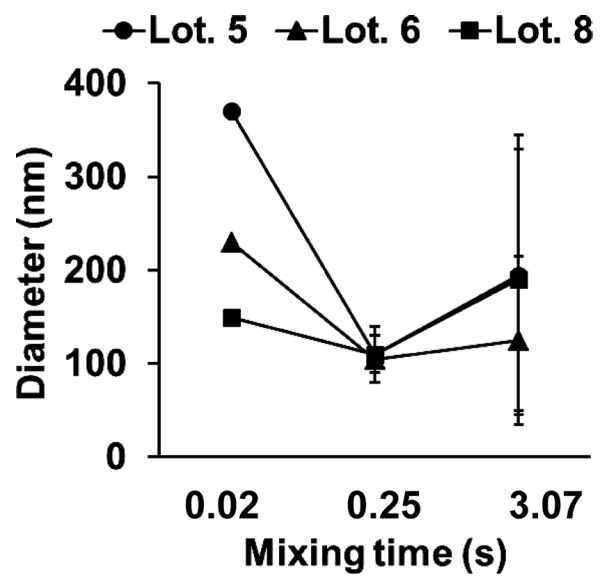

Fig. 6. The Effect of Mixing Time in the Type II Nozzle on the Size of EC Nanoparticles in MAN Microparticles

EC nanoparticle suspensions were prepared as described in Materials and Methods.

larger than that under the normal condition $(0.25 \mathrm{~s})$. Conversely, when the mixing time was increased $(3.07 \mathrm{~s})$, the mean diameter of EC nanoparticles was almost unchanged, but the standard deviation was much larger than that under the normal condition $(0.25 \mathrm{~s})$. These results suggest that optimizing the mixing time in the mixing component is necessary to produce EC nanoparticles in MAN microparticles. Decreasing the mixing time was insufficient to produce smaller EC nanoparticles. These experimental conditions may be similar to those of the four-fluid nozzle, ${ }^{6}$ although we cannot calculate the mixing time in the case of the four-fluid nozzle. As these solutions are mixed in the collision section, it is estimated that the mixing time for the drug-organic solvent solution and the aqueous solution is short when using the four-fluid nozzle. On the contrary, we assume that longer mixing times increase the time needed for formation of the drug-core, as indicated in Fig. 5, and/or that drug nano-cores combine with each other, and therefore, larger EC particles may be formed.

One of the significant findings of our present study was that nanoparticle-containing microparticles were produced in one step by the combination of an anti-solvent component and a spray dryer by using a two-solution mixing type spray nozzle.
Some articles have reported the nano-sized crystallization of water-insoluble drugs by using the anti-solvent effect at a beaker scale (lab scale). ${ }^{15,16)}$ However, some types of drugs grow very fast in anti-solvent, and thus, it was difficult to produce nano-sized drug particles in the beaker using the anti-solvent method alone. In such a case, the spray nozzle that we developed could be more effective because these suspensions were spray-dried immediately and produced microparticles before crystallization began.

\section{CONCLUSION}

In conclusion, we developed a two-solution mixing type nozzle (Type II) to produce EC/MAN microparticles in a single step. The EC nanoparticles in MAN microparticles exhibited a small diameter $(<110 \mathrm{~nm})$ which was superior to that of the drug/MAN microparticles in our previous study that were produced using a four-fluid spray nozzle. The customizable mixing components in the Type II nozzle is also useful for adjusting the mixing condition and controlling the size of the EC nanoparticles. Although the detailed mechanism of EC nanoparticle formation using the Type II nozzle is still unclear, this nozzle is useful for the preparation of nanoparticle-containing microparticles, and these formulations might be effective for handling drug nanoparticles at the manufacturing scale.

Acknowledgments This research was supported in part by a Grant-in-Aid for Scientific Research (C) 22590040 from the Japan Society for the Promotion of Science, and a Grant from the Mochida Memorial Foundation for Medical and Pharmaceutical Research.

\section{REFERENCES}

1) Merisko-Liversidge E, Liversidge GG. Nanosizing for oral and parenteral drug delivery: a perspective on formulating poorly-water soluble compounds using wet media milling technology. Adv. Drug Deliv. Rev., 63, 427-440 (2011).

2) Merisko-Liversidge E, Liversidge GG, Cooper ER. Nanosizing: a formulation approach for poorly-water-soluble compounds. Eur. J. Pharm. Sci., 18, 113-120 (2003).

3) Bailey MM, Berkland CJ. Nanoparticle formulations in pulmonary 
drug delivery. Med. Res. Rev., 29, 196-212 (2009).

4) Rogueda PG, Traini D. The nanoscale in pulmonary delivery. Part 1: Deposition, fate, toxicology and effects. Expert Opin. Drug Deliv., 4, 595-606 (2007).

5) Rogueda PG, Traini D. The nanoscale in pulmonary delivery. Part 2: Formulation platforms. Expert Opin. Drug Deliv., 4, 607-620 (2007).

6) Ohashi K, Kabasawa T, Ozeki T, Okada H. One-step preparation of rifampicin/poly(lactic-co-glycolic acid) nanoparticle-containing mannitol microspheres using a four-fluid nozzle spray drier for inhalation therapy of tuberculosis. J. Control. Release, 135, 19-24 (2009).

7) Mizoe T, Ozeki T, Okada H. Application of a four-fluid nozzle spray drier to prepare inhalable rifampicin-containing mannitol microparticles. AAPS PharmSciTech, 9, 755-761 (2008).

8) Mizoe T, Ozeki T, Okada H. Preparation of drug nanoparticle-containing microparticles using a 4-fluid nozzle spray drier for oral, pulmonary, and injection dosage forms. J. Control. Release, 122, 10-15 (2007).

9) Mizoe T, Beppu S, Ozeki T, Okada H. One-step preparation of drug-containing microparticles to enhance the dissolution and absorption of poorly water-soluble drugs using a 4-fluid nozzle spray drier. J. Control. Release, 120, 205-210 (2007).

10) Ozeki $T$, Beppu S, Mizoe $T$, Takashima Y, Yuasa H, Okada H. Preparation of polymeric submicron particle-containing microparticles using a 4-fluid nozzle spray drier. Pharm. Res., 23, 177-183 (2006).

11) Ozeki T, Beppu S, Mizoe $T$, Takashima Y, Yuasa H, Okada H. Preparation of two-drug composite microparticles to improve the dissolution of insoluble drug in water for use with a 4-fluid nozzle spray drier. J. Control. Release, 107, 387-394 (2005).

12) Fages J, Lochard H, Letournneau J, Sauceau M, Rodier E. Particle generation for pharmaceutical applications using supercritical fluid technology. Powder Technology, 141, 219-226 (2004).

13) Patomchaiviwat V, Paeratakul O, Kulvanich P. Formation of inhalable rifampicin-poly(L-lactide) microparticles by supercritical anti-solvent process. AAPS PharmSciTech, 9, 1119-1129 (2008).

14) Rodrigues M, Li J, Padrela L, Almeida A, Matos H, Azevedo E. Anti-solvent effect in the production of lysozyme nanoparticles by supercritical fluid-assisted atomization processes. J. Supercrit. Fluids, 48, 253-260 (2009).

15) Perrut M, Jung J, Leboeuf F. Enhancement of dissolution rate of poorly-soluble active ingredients by supercritical fluid processes. Part I: Micronization of neat particles. Int. J. Pharm., 288, 3-10 (2005).

16) Duarte AR, Roy C, Vega-González A, Duarte CM, Subra-Paternault $\mathrm{P}$. Preparation of acetazolamide composite microparticles by supercritical anti-solvent techniques. Int. J. Pharm., 332, 132-139 (2007). 